INFLAMMATION AND MOTILITY

\title{
COX-2 dependent inflammation increases spinal Fos expression during rodent postoperative ileus
}

\author{
C Kreiss, L A Birder, S Kiss, M M VanBibber, A J Baver
}

Gut 2003;52:527-534

\begin{abstract}
Background and aims: Cyclooxygenase 2 (COX-2) and prostaglandins (PGs) participate in the pathogenesis of inflammatory postoperative ileus. We sought to determine whether the emerging neuronal modulator COX-2 plays a significant role in primary afferent activation during postoperative ileus using spinal Fos expression as a marker.

Methods: Rats, and COX $-2^{+/+}$and $\mathrm{COX}-2^{-/-}$mice underwent simple intestinal manipulation. The effect of intestinal manipulation on Fos immunoreactivity (IR) in the $L_{5}-S_{1}$ spinal cord, in situ circumference, and postoperative leucocytic infiltrate of the intestinal muscularis was measured. Postoperative $\mathrm{PGE}_{2}$ production was measured in peritoneal lavage fluid. The dependence of these parameters on COX-2 was studied in pharmacological (DFU, Merck- Frosst, selective COX-2 inhibitor) and genetic (COX-2 ${ }^{-1-}$ mice) models.

Results: Postoperative Fos IR increased 3.7-fold in rats and 2.2-fold in mice. Both muscularis leucocytic infiltrate and the circumference of the muscularis increased significantly in rats and $\mathrm{COX}-2^{+/+}$mice postoperatively, indicating dilating ileus. Surgical manipulation markedly increased $\mathrm{PGE}_{2}$ levels in the peritoneal cavity. DFU pretreatment and the genetic absence of $\mathrm{COX}-2^{-/-}$prevented dilating ileus, and leucocytic infiltrate was diminished by $40 \%$ with DFU and by $54 \%$ in COX-2 $2^{-/}$mice. DFU reversed postsurgical intra- abdominal $\mathrm{PGE}_{2}$ levels to normal. Fos IR after intestinal manipulation was attenuated by approximately $50 \%$ in DFU treated rats and in COX-2 $2^{-/-}$mice.

Conclusions: Postoperatively, small bowel manipulation causes a significant and prolonged increase in spinal Fos expression, suggesting prolonged primary afferent activation. COX-2 plays a key role in this response. This activation of primary afferents may subsequently initiate inhibitory motor reflexes to the gut, contributing to postoperative ileus.
\end{abstract}

See end of article for authors' affiliations

Correspondence to:

Dr A J Baver, Division of

P toperative ileus remains an almost universal consequence of abdominal surgery leading to significant morbidity and patient discomfort, which prolongs hospitalisation and thus adds markedly to healthcare costs. Inflammatory and neuronal mechanisms have been implicated in postoperative dysmotility but the sequence of these events and their relative contribution to the pathogenesis remain poorly understood. Neural pathways, including spinal reflexes, ${ }^{23}$ neuropeptides, ${ }^{4}$ nitric oxide, ${ }^{5}$ and adrenergic receptors, ${ }^{67}$ have been found to play an important role in the development of this disorder. Our laboratory has focused on local inflammatory mechanisms within the intestine, showing in both humans and rodents that surgical manipulation of the gut leads to a marked molecular and cellular inflammatory response within the intestinal muscularis. ${ }^{58-10}$ The resulting inflammatory response has been shown to be proportional to the degree of gut ileus, as demonstrated by a decrease in gastrointestinal transit and suppression of in vitro circular smooth muscle contractility. We have also shown that leucocyte adhesion molecule blocking antibodies prevent the recruitment of monocytes, neutrophils, and mast cells into the muscularis and also avert postoperative jejunal muscle dysfunction. ${ }^{11}$ This observation underlines the importance of inflammatory changes within the intestinal muscularis in the aetiology of postoperative ileus. More recently, it has been demonstrated that leucocyte derived inducible nitric oxide synthase (iNOS) and cyclooxygenase 2 (COX-2) play a significant role in the pathogenesis of rodent postoperative ileus. ${ }^{51213}$ It was demonstrated that intestinal manipulation induces iNOS and COX-2 messenger RNA and protein within resident muscularis macrophages and recruited monocytes. Pharmacological inhibition or genetic manipulation of either iNOS or COX-2 resulted in improved postoperative in vitro jejunal circular muscle contractility and prevention of the postsurgical delay in gastrointestinal transit.

Various products released from leucocytes have been demonstrated to sensitise or even directly activate intestinal primary afferent neurones. Reactive radicals, such as superoxide, peroxynitrite, and hydrogen peroxide, generated by leucocytes during inflammation or ischaemia, ${ }^{14}$ have been shown to stimulate afferent splanchnic C fibre units. ${ }^{15}$ More recently, prostaglandins, secreted by various leucocyte populations during inflammation, ${ }^{14}$ have been shown to enhance primary afferent nerve firing. In particular, prostaglandin $\mathrm{E}_{2}$ $\left(\mathrm{PGE}_{2}\right)$ has been demonstrated to have complex effects on primary intestinal afferents. ${ }^{16-18}$ Cyclooxygenases are key enzymes in the production of prostaglandins, catalysing the conversion of arachidonic acid to prostaglandins (PG) $\mathrm{G}_{2}$ and $\mathrm{H}_{2}$. Two COX isoforms have been identified and are referred to as COX-1 and COX-2. While COX-1 is produced constitutively, COX-2 is a highly inducible enzyme, known to be triggered in many inflammatory states. ${ }^{19}$ COX-2 has been shown to be induced by interleukin 1 , bacterial lipopolysaccharide, and growth factors. ${ }^{20}$ Macrophages, one of the main leucocyte populations involved during inflammation of the muscularis after gut manipulation," express large increases in numerous proinflammatory cytokines, nitric oxide, and PGs. ${ }^{14}$ Recently, we have shown that PGs, through induction of COX-2, play a

Abbreviations: COX-2, cyclooxygenase 2; MPO, myeloperoxidase; $P G$, prostaglandin; $K O$, knockout; $M D H$, medial dorsal horn; $L D H$, lateral dorsal horn; DCM, dorsal commissure; SPN, sacral parasympathetic nucleus; IR, immunoreactivity; iNOS, inducible nitric oxide synthase. 
major role in causing postoperative ileus. ${ }^{12}$ Our data demonstrate that COX-2 inhibitors significantly improved the postsurgical suppression in small intestinal motility.

Fos is a nuclear phosphoprotein product of the immediate early gene Fos which may be used as a specific reproducible marker to map functional excitatory pathways in the central nervous system. ${ }^{21}$ We hypothesise that upregulation of COX-2 and subsequently PG production within the intestinal wall after intestinal manipulation will increase spinal Fos expression suggesting stimulation of primary afferents, thus increasing their activity and possibly enhancing inhibitory motor reflexes to the gut. This hypothesis is based on experimental data obtained from other gastrointestinal inflammatory models which have shown that inflammation of the gut wall stimulates afferent pathways to the spinal cord using Fos expression as a marker. ${ }^{22-24}$ To investigate this hypothesis, we determined the effect of intestinal manipulation on activation of primary intestinal afferents by measuring Fos-like immunoreactivity (IR) in the lumbosacral spinal cord. Next we studied events within the gut wall that could contribute to the increased activity of intestinal primary afferents. We determined the leucocytic infiltrate in the intestinal muscularis using myeloperoxidase (MPO) staining after intestinal manipulation and measured the in situ intestinal muscularis circumference. Subsequently, we quantified intra-abdominal release of $\mathrm{PGE}_{2}$ postoperatively. The dependence of these responses on COX-2 was then studied in pharmacological (5,5- dimethyl-3-(3-fluorophenyl)-4-(4-methylsulphonyl) phenyl-2(5H)-furan; DFU, Merck-Frosst, selective COX-2 inhibitor $)^{25}$ and genetic (COX-2 $2^{-/-}$mice) models of postoperative ileus.

\section{METHODS}

\section{Animals}

Sprague-Dawley male rats (220-300 g) were obtained from Harlan (Indianapolis, Indiana, USA). Homozygous wild-type C57BL/6 mice as well as homozygous COX-2- $2^{--}$knockout (KO) mice weighing 18-20 g were kindly provided by $\mathrm{Dr} \mathrm{SH}$ Graham (Department of Neurology, University of Pittsburgh, Pittsburgh, Pennsylvania, USA). The homozygous COX- $2^{-/-} \mathrm{KO}$ mice resulted from a cross of COX-2 $2^{+-}$heterozygote C57BL/6 mice (B6,129S- Ptgs $2^{\text {tm IJed; }}$ Jackson Laboratories, Bar Harbor, Maine, USA). ${ }^{26-28}$ The University of Pittsburgh Institutional Animal Care and Use Committee approved all experimental animal protocols. Animals were housed in a pathogen free facility that is accredited by the American Association for Accreditation of Laboratory Animal Care and complies with the requirements of humane animal care, as stipulated by the United States Department of Agriculture and the Department of Health and Human Services. They were maintained on a 12 hour light/dark cycle and provided with commercially available chow and tap water ad libitum. Genotypes of mice were determined on DNA isolated from tail clippings, using previously described protocols. ${ }^{27}$

\section{Experimental groups and operative procedures}

The small bowel of the animals was subjected to an easily standardised mild surgical manipulation, as described previously. ${ }^{9}$ Unoperated animals served as corresponding controls. In brief, before the beginning of surgery, animals were anaesthetised with isoflurane inhalation and a midline incision was made into the peritoneal cavity. The small bowel was eventrated to the left onto moist gauze, and the entire small bowel was lightly manipulated between two moist cotton applicators. After manipulation, laparotomy of each animal was closed using a double layer running suture. The bowel manipulation procedure caused no mortality. Animals recovered quickly from surgery and generally began to eat and drink within two hours. For this study, animals were sacrificed 24 hours after manipulation. This time point was chosen as it has been previously shown that the postoperative leucocyte infiltrate in the intestinal muscularis is fully developed. ${ }^{29} \mathrm{We}$ also chose this later time point as we wanted to study specifically the interaction of postoperative inflammation with intestinal primary afferents, without any immediate interference from possible mechanical stimulation of primary intestinal afferents by the surgical procedure itself. The highly selective COX-2 inhibitor DFU (Merck-Frosst) was dissolved in DMSO and administered at $10 \mathrm{mg} / \mathrm{kg}$ subcutaneously, 30 minutes before intestinal manipulation and again four hours postoperatively. The rat experiments included four groups of animals (each $n=4-6$ ): controls; controls treated with DFU; rats undergoing intestinal manipulation; and rats undergoing intestinal manipulation treated with DFU. For experiments measuring Fos IR in the spinal cord, a fifth experimental group was added consisting of animals undergoing laparotomy only without intestinal manipulation $(n=4)$. The mouse experiments consisted of four experimental groups: $\mathrm{COX}_{-} 2^{+/+}$and $\mathrm{COX}-2^{-/-}$mice underwent small bowel intestinal manipulation. Unoperated COX $-2^{+/+}$and $\mathrm{COX}-2^{-/-}$mice were used as controls.

\section{Histochemistry stainings for MPO activity and measurement of the in situ circumference of the intestinal muscularis}

Whole mounts of the intestinal muscularis from paraformaldehyde perfused animals were investigated for the presence of resident and recruited leucocytes. Mid jejunal segments approximately $3-4 \mathrm{~cm}$ in length were cut from the bowel and immersed in chilled KRB in a Sylgard bottom coated glass dish (Dow-Corning, Midland, Michigan, USA). The segment was gently pinned down along the mesenteric border. The bowel was opened along the mesentery and washed twice in Krebs buffer, and mucosa and submucosa were stripped off under microscopic observation (Wild-M8, Heerbrugg, Switzerland). The mucosa free muscularis whole mounts were used for staining procedures. Polymorphonuclear neutrophils were visualised by a MPO stain: freshly prepared whole mounts were immersed in a mixture of $10 \mathrm{mg}$ Hanker-Yates reagent (Sigma Chemical Co, St Louis, Missouri, USA), $10 \mathrm{ml} \mathrm{KRB,} \mathrm{and}$ $100 \mu \mathrm{l} 3 \%$ hydrogen peroxide (Sigma) for 10 minutes. The reaction was stopped with cold Krebs. Whole mounts were cover slipped and inspected by light microscopy after staining (Nikon FXA; Fryer, Huntley, Illinois, USA). Leucocytes were counted in five randomly chosen areas in each specimen at a magnification of 200x. The in situ circumference of the muscularis was measured by determining the greatest width of each muscularis whole mount with a caliper.

\section{Measurement of $\mathrm{PGE}_{2}$ in peritoneal lavage fluid}

Intra-abdominal release of $\mathrm{PGE}_{2}$ was determined in controls and in rats 24 hours after intestinal manipulation with and without DFU treatment. The peritoneal cavities of these two groups were injected with $4 \mathrm{ml}$ of warmed sterile isotonic sodium chloride solution (Abbott Laboratories, North Chicago, Illinois, USA) and the animal was then sacrificed after a period of 90 minutes. At the time of sacrifice the peritoneal liquid was aspirated and the concentration of $\mathrm{PGE}_{2}$ was measured by ELISA (Amersham Pharmacia Biotech Inc., Piscataway, New Jersey, USA).

\section{Measurement of Fos immunoreactivity (IR)}

Twenty four hours after intestinal manipulation, animals were anaesthetised (pentobarbital $50 \mathrm{mg} / \mathrm{kg}$ intraperitoneally) and sacrificed via intracardiac perfusion first with Kreb's buffer followed by $4 \%$ paraformaldehyde fixation. The spinal cord $L_{5}-S_{1}$ was removed and postfixed overnight in the same fixative at $4^{\circ} \mathrm{C}$. Tissues were subsequently cryoprotected in 30\% sucrose solution overnight at $4^{\circ} \mathrm{C}$ and embedded in OCT embedding medium (Tissue- Tek; Sakura Finetek Inc., Torrance, California, 


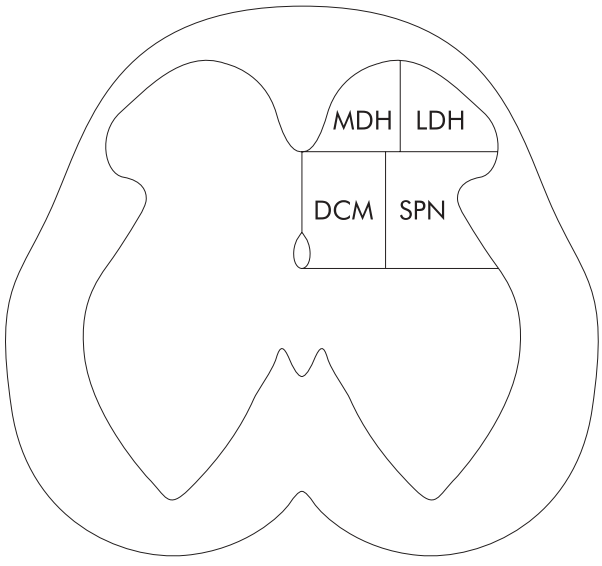

Figure 1 Drawing of a section from the $\mathrm{L}_{6}$ spinal cord in the rat depicting four regions where Fos positive neurones are located. $\mathrm{MDH}$, medial dorsal horn; $L D H$, lateral dorsal horn; DCM, dorsal commissure; and SPN, sacral parasympathetic nucleus.

USA). Alternate floating sections $(30 \mu \mathrm{m})$ of the spinal cord $\mathrm{L}_{5}-\mathrm{S}_{1}$ were processed for Fos IR, as described previously, ${ }^{3031}$ using an avidin-biotin method for the Fos protein with primary antibodies at a dilution of 1:60 000 (Calbiochem, La Jolla, California, USA) and goat antirabbit secondary antibodies at a dilution of 1:600 (Vector, Burlingame, California, USA).

Counts of Fos positive cells on one side of the spinal cord are presented as the cumulative mean of all three segments. The number of labelled neurones was estimated from counts of positively stained cells in a minimum of 10 sections per spinal cord segments. Sections used for the counts were separated by at least $100 \mu \mathrm{m}$ to eliminate counting of a neurone more than once. Cells exhibiting Fos protein IR were counted in four regions, similar to those described earlier, ${ }^{30-32}$ for assessing regional distribution: medial dorsal horn $(\mathrm{MDH})$, lateral dorsal horn (LDH) including the superficial laminae I and II, dorsal commissure (DCM) including lamina $\mathrm{X}$, and the sacral parasympathetic nucleus (SPN) including the lateral laminae V-VII (fig l). Analysis of the regional distribution of Fos IR was limited to the $\mathrm{L}_{6}$ segment, as this had the largest number of cells.

The $\mathrm{L}_{5}-\mathrm{S}_{1}$ segment of the spinal cord was chosen as it has previously been shown that primary intestinal afferents from the lower gastrointestinal tract project to this segment. ${ }^{23} 24334$ To confirm that the majority of neurones expressing Fos postoperatively were in the $\mathrm{L}_{5}-\mathrm{S}_{1}$ segments, we quantified Fos expression within the spinal cord segments $\mathrm{T}_{10}-\mathrm{S}_{2}$ in two animals postoperatively.

\section{Calculations and statistics}

Data are presented as mean (SEM). Changes in the in situ circumference of the intestinal muscularis, MPO positive cells, intra-abdominal $\mathrm{PGE}_{2}$ concentration, and Fos IR were evaluated statistically by ANOVA followed by a Scheffe multiple comparison test using STATA software (STATA corporation, College Station, Texas, USA). Differences in regional distribution of Fos IR between manipulated rats with/without DFU pretreatment and between manipulated COX $-2^{+/+}$and COX-2 $2^{-+}$ mice were compared by an unpaired Student's $t$ test. Data were considered statistically significant at $\mathrm{p}<0.05$.

\section{RESULTS}

Intestinal manipulation causes a sustained upregulation of spinal cord Fos

We hypothesised that the inflammatory responses within the gut wall cause an increase in spinal Fos expression. To obtain evidence to support this hypothesis, we quantified Fos expression within spinal cord segments $\mathrm{T}_{10}-\mathrm{S}_{2}, 24$ hours after selective surgical manipulation of the rat small intestine in a

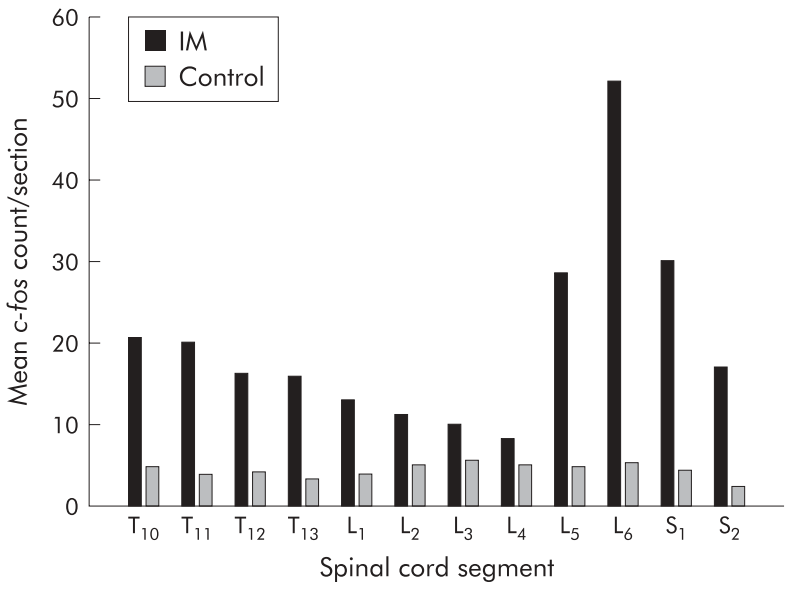

Figure 2 Histogram showing the segmental distribution of Fos positive cells (number/section) in a typical rat spinal cord from a control rat and from a rat after intestinal manipulation (IM) $\left(T_{10}-S_{2}\right)$

group of rats. These data showed that a significant increase in Fos-like IR was primarily localised to $\mathrm{L}_{5}-\mathrm{S}_{1}$ compared with spinal Fos-like IR of control rats. A typical example of the distributed increase in Fos positive spinal neurones of a manipulated animal is shown in fig 2. In control rats, baseline Fos IR cells cumulatively numbered 45.1 (5.71) for spinal cord segments $\mathrm{L}_{5}-\mathrm{S}_{1}$. Twenty four hours after intestinal manipulation, a 3.7- fold increase in Fos IR cells was quantified ( 168.0 (6.90); $\mathrm{p}<0.001$ ). Laparotomy alone did not have a significant effect on spinal Fos IR 24 hours postoperatively in rats (41.1 (10.6)), indicating that the increase in Fos positive cells in the manipulated animals was directly related to the sequelae of intestinal manipulation rather than to anaesthesia or laparotomy. The largest percentage of postoperative Fos IR was located in the LDH region $(37.2(4.71) \%)$, with smaller percentages in the MDH (22.8 (1.84)\%), DCM (22.1 (2.54)\%), and SPN, including the lateral laminae V-VII ( 17.9 (3.66)\%). In unmanipulated control rats, total numbers of Fos IR cells were low and regional differences were not significant (approximately 5-10 Fos positive cells per section).

\section{Manipulation induced intestinal circumferential dilatation and leucocyte infiltration}

Next, we sought to determine events within the gut wall that could contribute to increased spinal Fos expression. Bowel wall distension is known to activate intestinal afferents ${ }^{23}$ and typical flat plate abdominal radiographs of patients suffering from postoperative ileus show a dilated bowel. ${ }^{35}$ As shown in fig $3 \mathrm{~A}$ and $3 \mathrm{~B}$, this clinical observation was recapitulated in our model of postoperative ileus such that compared with controls, intestinal manipulation caused a significant increase in intestinal muscularis in situ circumference in both rats (controls $0.9(0.1) \mathrm{cm}$; intestinal manipulation $1.3(0.1) \mathrm{cm}$ ) and wild-type COX-2 $2^{+/+}$mice (controls 0.85 (0.1) cm: intestinal manipulation $1.3(0.1) \mathrm{cm})$. Laparotomy in itself did not affect rat intestinal 24 hour postoperative in situ circumferences $(1.0(0.1) \mathrm{cm})$.

The manipulation induced increase in bowel wall circumference was associated with an increase in MPO positive $\left(\mathrm{MPO}^{+}\right)$cells within the muscularis. These types of leucocytes are known to secrete substances which are known to sensitise primary afferents ${ }^{8}$ and contribute to bowel dysmotility. ${ }^{29}$ In control rodents, low numbers of $\mathrm{MPO}^{+}$cells were quantified within the intestinal muscularis (rat: 2.7 (0.34) cells/field; mice: 0.6 (0.27) cells/field at $200 \times$ magnification). However, as shown in figs 4 and 5, intestinal manipulation resulted in a significant extravasation of $\mathrm{MPO}^{+}$cells into the muscularis of both rats and $\mathrm{COX}-2^{+/+}$mice (rats: 92.6 (12.10) cells/field; COX-2 $2^{+/+}$mice: 58.7 (9.40) cells/field at $200 \times$ magnification). 

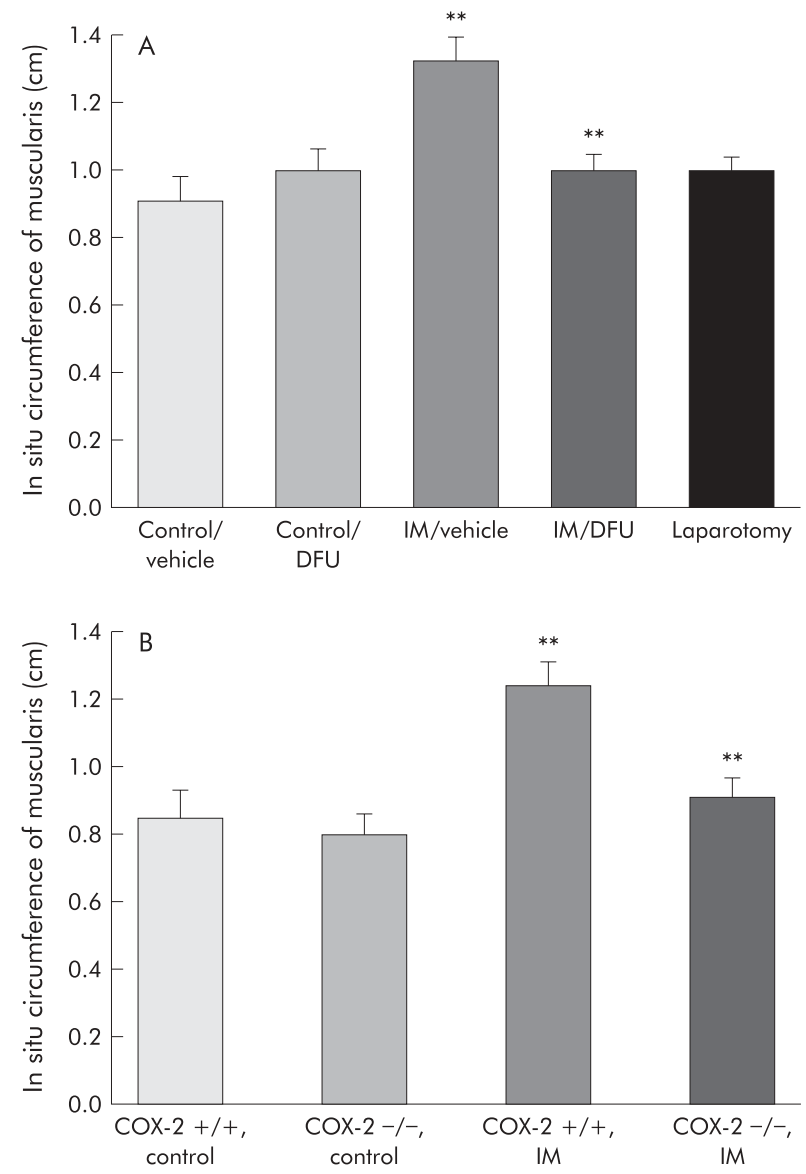

Figure 3 Histogram showing the in situ circumference of the intestinal muscularis in (A) control rats with and without DFU, in surgically manipulated (IM) rats with and without DFU, and in rats undergoing laparotomy only, and in (B) COX $-2^{+/+}$and $\mathrm{COX}-2^{--}$ control mice and $\mathrm{COX}-2^{+/+}$and $\mathrm{COX}-2^{-/-}$surgically manipulated (IM) mice. Surgical manipulation caused a marked increase in the in situ muscularis circumference indicating dilating ileus $(\mathrm{p}<0.008)$. This effect was significantly attenuated in both DFU treated rats and COX-2 $2^{-/-}$mice. $n=4-5 ;{ }^{*} \mathrm{p}<0.02$.

\section{Pharmacological (DFU) and genetic $\left(\mathrm{COX}-2^{-1-}\right)$ \\ modulation of COX-2}

We have previously demonstrated that the inducible COX-2 pathway plays a significant role in postoperative dysmotility. ${ }^{12}$ Here we show that pharmacological and genetic manoeuvres to limit COX-2 activity significantly prevent intestinal dilation caused by intestinal manipulation. Manipulated rats pretreated with DFU $(10 \mathrm{mg} / \mathrm{kg})$ and manipulated $\mathrm{COX}-2^{-/-}$mice did not manifest bowel dilatation and had intestinal circumferences that were comparable with control unmanipulated rodents, as shown above (DFU treated manipulated rats: $1.0(0.1) \mathrm{cm}$; COX-2 ${ }^{-/}$manipulated mice: $0.9(0.1) \mathrm{cm} ; \mathrm{n}=5$ for all groups) (fig 3). DFU administered to control unoperated animals did not alter intestinal circumference $(1.0(0.04) \mathrm{cm})$ compared with control animals.

Just as manipulation induced dilatation was associated with a strong leucocytic infiltrate, as mentioned above, prevention of dilatation through pharmacological and genetic manipulation of COX-2 was associated with a decrease in leucocyte infiltration into the intestinal muscularis. DFU treated controls or laparotomy alone did not alter the baseline number of $\mathrm{MPO}^{+}$cells quantified in the muscularis whole mounts (DFU treated controls: $2.1(0.16)$ cells/field at $200 \times$ magnification; laparotomy only: 2.8 (0.31) cells/field) compared with unoperated controls. Likewise, $\mathrm{MPO}^{+}$cells were found in low numbers in COX $-2^{-/-}$mice within the muscularis $(0.7(0.37)$ cells/field). As shown in figs 4 and 5 , the intestinal
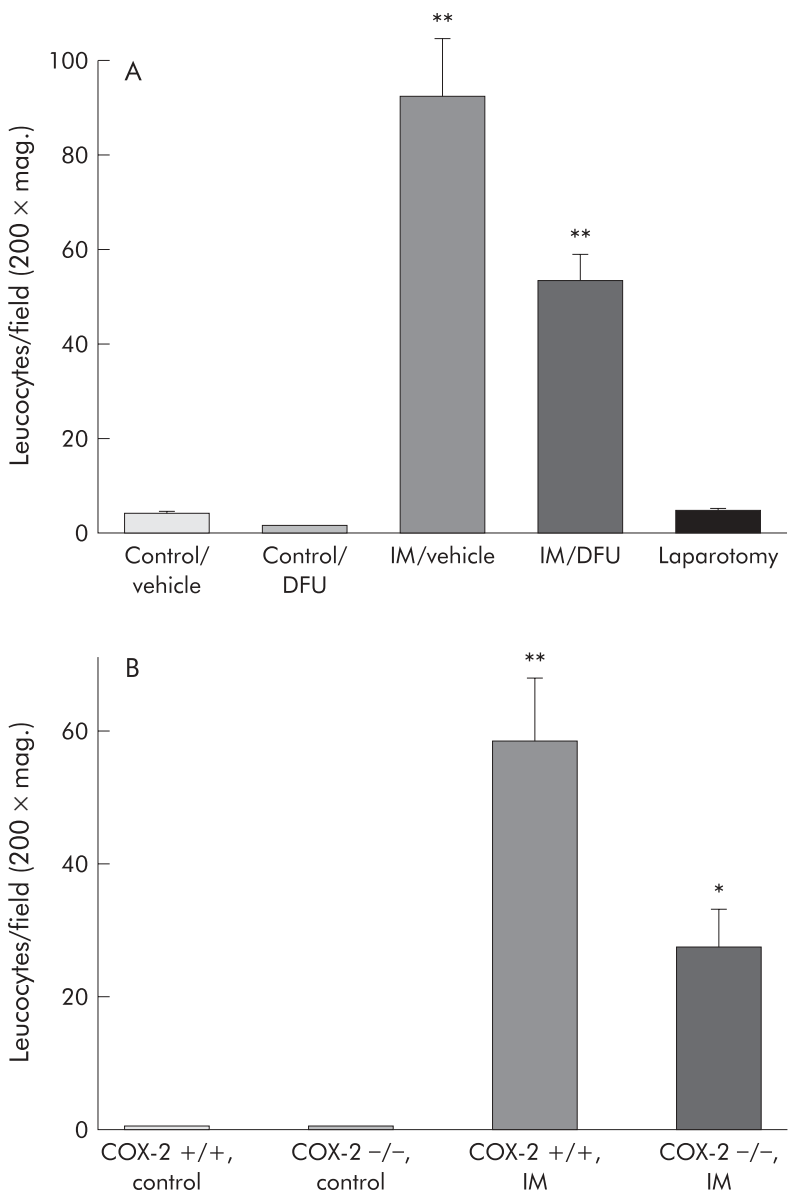

Figure 4 Histogram of infiltrating leucocytes in muscularis whole mounts from (A) control rats with and without DFU, from surgically manipulated (IM) rats with and without DFU, and from rats

undergoing laparotomy only and from (B) COX $-2^{+/+}$and $\mathrm{COX}-2^{-/-}$ control mice and COX-2 $2^{++}$and COX-2 $2^{-/-}$surgically manipulated (IM) mice. Surgical manipulation caused a significant increase in leucocytic infiltrate $(p<0.008)$, which was markedly diminished in both DFU treated rats and COX- $2^{-1-}$ mice. $n=4-5 ;{ }^{* *} p<0.02$.

manipulation induced extravasation of leucocytes into the rat intestinal muscularis was significantly prevented with DFU pretreatment by $40 \%$ (53.3 (5.79) cells/field). Similarly, COX-2 deficient mice showed a significant $54 \%$ decrease in $\mathrm{MPO}^{+}$ muscularis leucocytic infiltrates after intestinal manipulation (27.2 (5.90) cells/field) compared with manipulated wild-type mice.

\section{DFU prevents the postoperative intra-abdominal production of $\mathrm{PGE}_{2}$}

$\mathrm{PGE}_{2}$ is produced by inflammatory cells during inflammation and has been demonstrated to be one of the important factors which sensitise intestinal primary afferents. ${ }^{17}$ Intestinal manipulation caused a 3.1-fold increase in $\mathrm{PGE}_{2}$ concentration measured in the intraperitoneal lavage fluid of rats 24 hours after surgery (controls $213.3(26.4) \mathrm{pg} / \mathrm{ml}$; intestinal manipulation 669.0 (62.9) $\mathrm{pg} / \mathrm{ml} ; \mathrm{p}<0.003$ ) (fig 6). Selective inhibition of COX-2 with DFU did not significantly alter baseline intra-abdominal $\mathrm{PGE}_{2}$ levels measured from peritoneal lavage fluid (200.8 (16.1) pg/ml) but DFU markedly decreased the observed postsurgical increase in $\mathrm{PGE}_{2}$ in the abdomen (260.8 (20.0) pg/ml) after manipulation. Figure 6 illustrates that postoperative $\mathrm{PGE}_{2}$ concentrations in the peritoneal lavage fluid were maintained at baseline levels in both DFU treated groups of animals $(\mathrm{p}<0.006)$. 

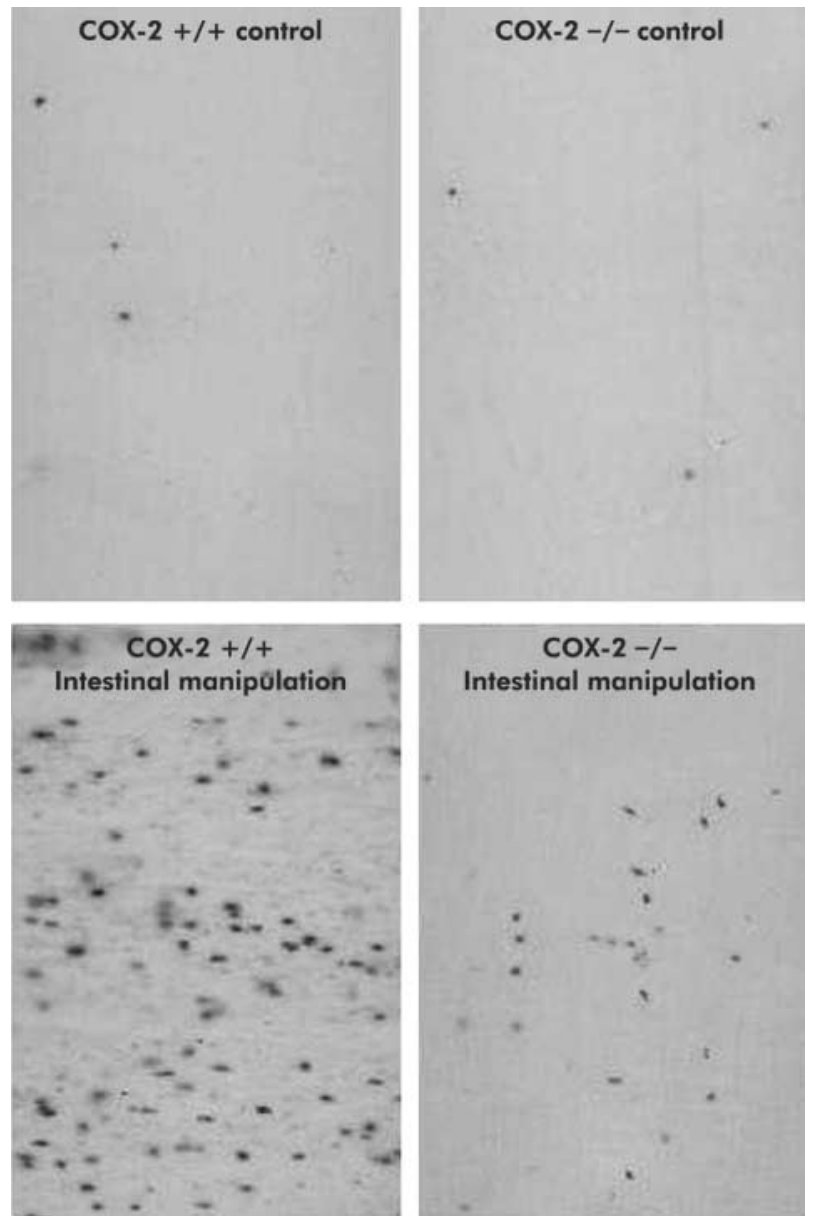

Figure 5 Myeloperoxidase (MPO) staining of jejunal muscularis whole mounts in mice after intestinal manipulation. A low number of MPO positive cells was seen in both COX-2 $2^{+/+}$and COX $-2^{-1}$

controls. Surgical manipulation caused a significant increase in MPO positive cells that was attenuated in COX $-2^{-/-}$mice.

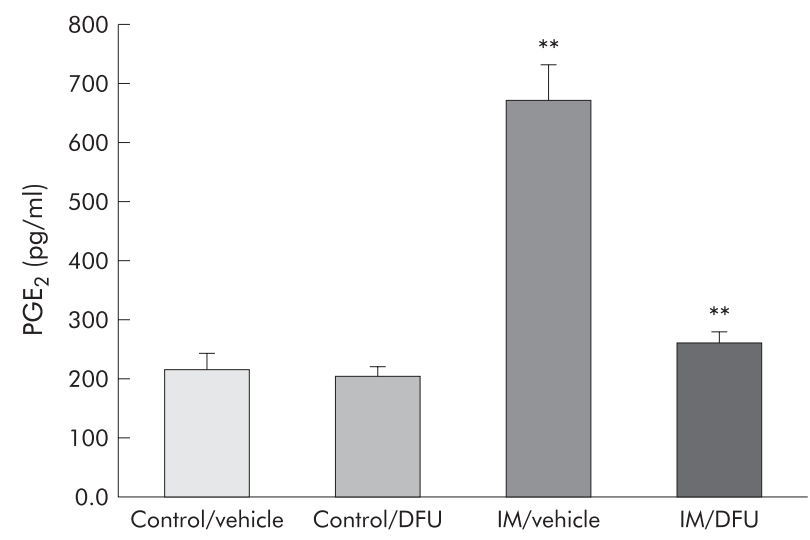

Figure 6 Histogram demonstrating a significant increase in prostaglandin $\mathrm{E}_{2}\left(\mathrm{PGE}_{2}\right)$ levels in peritoneal lavage fluid measured 24 hours after intestinal manipulation (IM) using ELISA. This increase was reversed to control levels after administration of DFU. $n=6$; ${ }^{* *} p<0.006$.

\section{Pharmacological (DFU) and genetic (COX-2 ${ }^{-1}$ ) modulation of COX-2 significantly limits the rise in postoperative spinal cord Fos expression}

Finally, we sought to determine if the postoperative COX-2 sensitive responses to intestinal manipulation (that is, bowel dilatation, inflammatory infiltrate, and increased intraabdominal $\mathrm{PGE}_{2}$ levels) contributed to the observed sustained
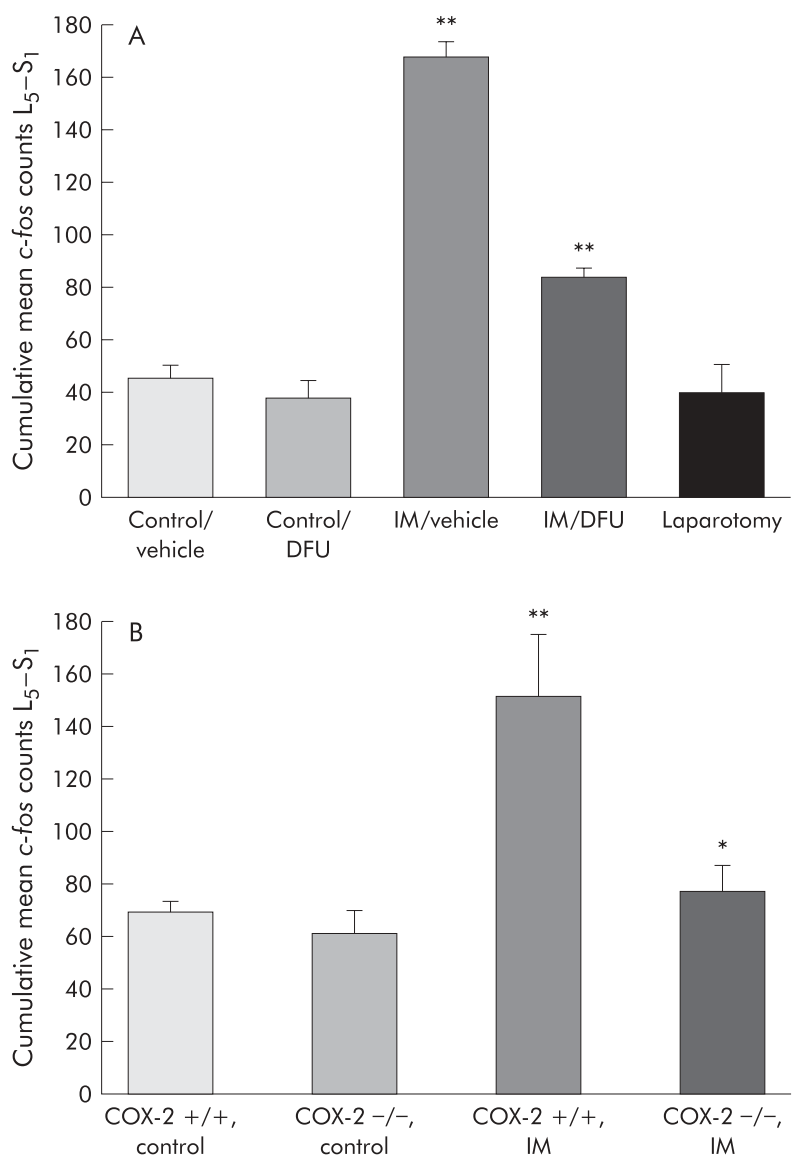

Figure 7 Histogram showing cumulative mean Fos counts in (A) control rats with and without DFU, in surgically manipulated (IM) rats with and without DFU, and in rats undergoing laparotomy only, and in (B) COX $-2^{+/+}$and COX $-2^{-/-}$control mice and COX $-2^{+/+}$and COX $-2^{-/-}$surgically manipulated (IM) mice. Surgical manipulation caused a significant increase in postoperative Fos expression $(p<0.008)$ which was markedly decreased in both DFU treated rats and COX $-2^{-/-}$mice. $n=4-5 ;{ }^{*} p<0.02$.

increase in postoperative Fos expression within $\mathrm{L}_{5}-\mathrm{S}_{1}$ spinal cord neurones. As shown in figs 7 and 8, pharmacological blockade of COX-2 with DFU did not alter baseline numbers of Fos IR cells (controls 45.1 (5.71) cumulative mean Fos counts $\mathrm{L}_{5}-\mathrm{S}_{1}$; DFU treated controls 38.0 (7.36)). However, DFU significantly attenuated by $50 \%$ the 3.7 -fold increase in Fos IR cells induced by intestinal manipulation (control manipulation 167.90 (6.90) $v$ DFU manipulation 84.3 (3.60); $<<0.001$, $\mathrm{n}=5$ each). DFU pretreatment markedly reduced the percentage of Fos IR cells in the LDH $(25.5(3.17) \%$; $<<0.05)$ while the distribution in the MDH (24.6 (2.36)\%), DCM (29.3 (2.65\%), and SPN (20.6 (3.26)\%) were not significantly affected. Figure 8 shows the typical distribution of Fos IR positive cells in four groups of animals (control, manipulated, manipulated with DFU pretreatment, and a laparotomised rats).

Similarly, genetically deficient mice also showed attenuated Fos activation compared with $\mathrm{COX}-2^{+/+}$wild-type mice. Although baseline Fos IR spinal cord cell counts were similar between $\mathrm{COX}^{+/+}\left(69.0\right.$ (4.3) cells) and COX-2 $2^{-/}$mice (61.6 (8.3) cells), manipulated COX- $2^{-/}$mice exhibited $49 \%$ fewer Fos IR neurones ( 77.9 ( 10.3 cells) compared with manipulated wildtype mice (152.0 (23.30) cells) (fig 7B). No regional predominance in Fos IR in manipulated $\mathrm{COX}^{+/+}$mice could be distinguished (LDH 26.8 (2.35)\%, MDH 23.6 (5.37)\%, DCM $25.5(1.86) \%$, SPN including the lateral laminae V-VII 24.2 (3.81)\%). Additionally, Fos IR was decreased overall in manipulated $\mathrm{COX}-2^{-/}$mice without a significant preferential decrease in any particular area. 

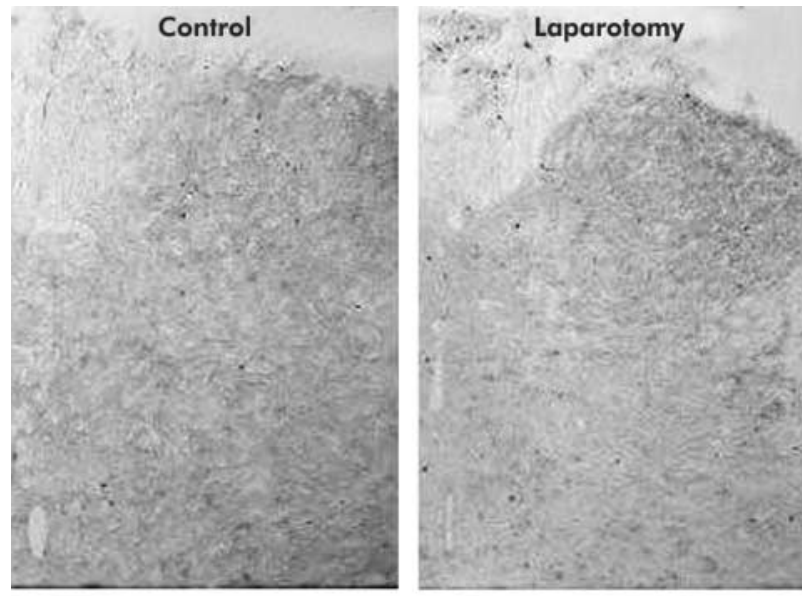

Intestinal manipulation
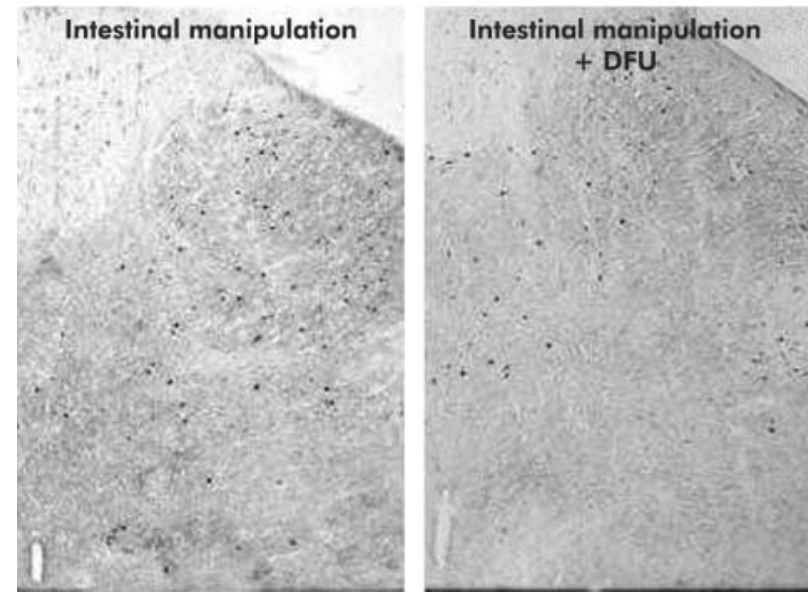

Figure 8 Representative images illustrating distribution of Fos IR in the $L_{6}$ spinal cord of a control rat, a surgically manipulated rat, a surgically manipulated rat after DFU treatment, and of a rat that underwent laparotomy only.

\section{DISCUSSION}

In the present study, we have shown for the first time that COX-2 dependent inflammatory responses play an important role in long term postoperative spinal Fos expression after surgical manipulation of the small intestine. Previous studies have clearly identified the importance of both a neurogenic and a local inflammatory mechanism for causing postoperative ileus. Our current demonstration of the immunological modulation of spinal Fos expression, used as a marker for activation of visceral afferents, creates a mechanistic link between the postoperative inflammatory events that we have previously delineated within the intestinal muscularis ${ }^{5} 9111229$ and an inhibitory neuronal reflex that participates in causing postoperative ileus.

Noxious distension, inflammation or chemical irritation of the gastrointestinal tract, ${ }^{22-2433}$ urinary bladder, ${ }^{30}{ }^{31}$ and skin $^{37}$ have been used to stimulate and map afferent pathways to the spinal cord using Fos expression. These models have been shown to "sensitise" polymodal visceral sensory fibres by reducing their threshold for activation and by increasing their spontaneous and evoked firing rate..$^{38}$ Additionally, inflammation has been shown to awaken "silent afferent fibres". ${ }^{39}{ }^{40}$ Our data showing an increase in Fos-like IR within the spinal cord 24 hours following surgery suggest that intestinal manipulation, which frequently occurs in a clinical setting, initiates a series of events that results in a prolonged increase in the activation of intestinal primary afferent neurones. However, Fos expression remains an indirect measure to map primary afferent pathways to the spinal cord and several other possibilities need to be considered that could have contributed to the enhanced Fos expression. Limitations of the observed change in Fos expression include the possibility that Fos-like IR could also be centrally mediated through reduced descending inhibition, or through supraspinal or segmental activation.

The distribution of the manipulation enhanced inputs appeared to be predominantly localised to $\mathrm{L}_{5}-\mathrm{S}_{1}$ segments of the rat spinal cord. Only the lower small intestine is known to project to this area, while the proximal and mid small bowel projects to the lower thoracic segments. Fos counts were elevated in the $T_{10}-T_{12}$ segments, but to a lesser degree than in the $\mathrm{L}_{5}-\mathrm{S}_{1}$ segments. It is thus possible that afferents from secondary events in the colon, which project mainly to the lumbosacral segments, ${ }^{41}$ could account for a large portion of the observed change. We have recently shown that selective jejunal manipulation also compromises colonic circular muscle contractility and causes significant upregulation in several inflammatory mediators, including COX-2, in both the gastric and colonic muscularis. ${ }^{42}$ Thus PG synthesis appears to play an important role in the postsurgical increase in Fos expression in the $\mathrm{L}_{5}-\mathrm{S}_{1}$ spinal cord after intestinal manipulation. Neutrophils were also significantly recruited into the gastric and colonic muscularis after selective jejunal manipulation. It is thus conceivable that this panenteric "field effect" plays an important role in why Fos expression is mostly increased in the $\mathrm{L}_{5}-\mathrm{S}_{1}$ spinal cord after intestinal manipulation. Clinically, this is also an important observation as colonic ileus is known to occur in patients after selective gastric or small intestinal surgery. ${ }^{43}$ The effective duration of ileus is also mainly dependent on the return of colonic motility as the average postsurgical paralytic state lasts the longest in the colon. ${ }^{1}$ Within the $\mathrm{L}_{5}-\mathrm{S}_{1}$ spinal segments the largest percentage of postoperative induced Fos IR was located in the LDH, with smaller and relatively equal percentages distributed in the MDH, DCM, and SPN. The spinal distribution of Fos expression after manipulation is similar to other pelvic visceral afferent mapping investigations which report a lumbosacral pattern of neuronal activation that is localised to the MDH, LDH, DCM, and SPN..$^{23}{ }^{44}$ The fact that we saw Fos expression not only in peripheral, but also deeper laminae, may reflect that at the 24 hour time point there is polysynaptic activation of neurones other than primary afferent neurones involved in processing information from the inflamed intestine. However, it has also been suggested that with ongoing peripheral inflammation Fos expression in the rat spinal cord moves from the more superficial laminae to deeper laminae. ${ }^{45}$ Together, these results suggest that neurones in several regions of the spinal cord are involved in processing information from afferent inputs of the inflamed intestine.

Visceral sensory nerves have previously been implicated in the development of postoperative ileus. Desensitisation of intestinal primary afferents with capsaicin pretreatment has been shown to reduce the postoperative delay in gastrointestinal transit ${ }^{2}$ and to increase postoperative motility. ${ }^{3}$ The neurotransmitter calcitonin gene related peptide, which is released from sensory neurones on stimulation, appears to be a component of this neuronal mechanism in causing postoperative gastric ${ }^{46}$ and colonic ${ }^{4}$ ileus. However, in all of these studies gastrointestinal transit or motility measurements were performed within a few hours after surgical manipulation of the gut. Thus it is possible that some of these observations could have been confounded by the immediate activation of mechanoreceptors through the procedure itself or by anaesthesia. The present study showing persistent elevation in spinal Fos expression suggests for the first time that primary intestinal afferents remain activated for at least a 24 hour period postoperatively when the direct effects of mechanical stimulation and anaesthesia have worn off. ${ }^{29}$ It has previously been shown that spinal Fos expression peaks after a stimulus at about 1-2 hours, and that the number of Fos positive cells declines after six hours returning to control levels 24 hours 
after the original stimulus. ${ }^{31}$ Thus the marked increase in spinal Fos-like IR at 24 hours indicates ongoing neuronal activation. This extended time point after surgery may reflect more closely the mechanisms that cause clinical postoperative ileus, which can last several days after surgery. ${ }^{1}$ In this study we have demonstrated activation of the afferent sensory arm, using Fos expression as a marker, but also propose that stimulation of primary intestinal afferents will subsequently activate inhibitory sympathetic reflexes to the gut contributing to the pathogenesis of postoperative intestinal muscle dysfunction. Autonomic adrenergic pathways have been shown to be involved in the pathogenesis of postoperative ileus $^{6747}$ as administration of alpha-2 adrenergic antagonists significantly decreased ${ }^{77}$ or even reversed ${ }^{6}$ postoperative intestinal motility disturbances.

As bowel distension itself is known to activate visceral afferent neurones ${ }^{48}$ and we observed postoperative dilatation of the bowel following surgery, bowel wall stretch could be one of the factors that contributed to the increase in Fos positive spinal neurones. It is very common for the physician to radiographically observe a significant circumferential enlargement in the postsurgical bowel in a clinical setting. ${ }^{35}$ We hypothesise that the increase in bowel diameter is mediated by the postsurgical ensuing inflammatory response, which is caused by bowel manipulation. This same inflammatory milieu is known to consist of mediators that have been hypothesised to mechanistically participate in the hyperalgesic response of the gut, which include: histamine, serotonin, tachykinins, prostanoids, cytokines, neurotrophins, and reactive intermediates. Also, many of these mediators are known to be derived from leucocytes. ${ }^{5} 12404950$ Based on evidence for direct involvement of prostanoids in sensitising intestinal primary afferents, ${ }^{16-18}$ in this study we directly measured intra-abdominal levels of $\mathrm{PGE}_{2}$ and our results quantified a significant increase in intraabdominal $\mathrm{PGE}_{2}$ levels following manipulation.

$\mathrm{PGE}_{2}$ has been found to have an inhibitory effect on gastrointestinal motility both in vivo ${ }^{51-53}$ and in vitro. ${ }^{54}$ While a direct inhibitory effect of PGs on jejunal circular muscle has been previously implicated by our findings, ${ }^{12}$ the present study provides evidence that endogenously produced PGs during postoperative ileus could additionally affect gastrointestinal motility through activation of primary afferents, as evidenced by an increase in spinal Fos expression. This possible activation of primary afferents would subsequently initiate inhibitory motor reflexes to the gut that contribute to postoperative ileus. A similar mechanism has previously been demonstrated in the bladder where it was found that endogenous $\mathrm{PGE}_{2}$ alters the efferent reflex limb of micturition by sensitising capsaicin sensitive afferents..$^{55}$

Pharmacological and genetic ablation of COX-2 significantly reduced the postoperative inflammatory leucocytic infiltrate within the intestinal muscularis. COX-2 inhibition could have decreased the inflammatory infiltrate by various mechanisms. Although beyond the scope of this investigation, we speculated that as endogenous $\mathrm{PGE}_{2}$ has been shown to stimulate interleukin 6 synthesis in macrophages ${ }^{56}$ and interleukin 6 has been shown to contribute to leucocyte recruitment during haemorrhagic shock, ${ }^{57}$ it is conceivable that COX- 2 blockade could attenuate the postoperative leucocytic infiltrate by decreased PGE, production, which will lead to diminished interleukin 6 levels and thus a decrease in leucocyte recruitment. Additionally, COX-2 inhibitors have also been shown to have anti- inflammatory effects independent of COX-2 activity-for example, through inhibiting proinflammatory transcription factors such as nuclear factor $\mathrm{\kappa B} .{ }^{58}$

Interestingly, anaesthesia and laparotomy together did not affect Fos IR, bowel circumference, muscularis leucocytic infiltrates, or PGE, levels, thus demonstrating that alterations in these parameters were due to the sequelae of intestinal manipulation rather than anaesthesia or laparotomy. In contrast, experiments in which COX-2 was pharmacologically blocked or was genetically absent demonstrated that bowel dilation, muscularis infiltration, and a subsequent increase in intra-abdominal $\mathrm{PGE}_{2}$ production were mediated by an increase in COX-2 synthase activity. Additionally, the similarity in the observations using pharmacological blockade of COX-2 and $\mathrm{COX}_{-2} 2^{-/-}$mice indicates that no compensatory pathways developed in $\mathrm{COX}-2^{-/}$mice in regard to this neuromodulatory function of COX-2

Others have shown previously that selective COX-2 inhibition can decrease spinal Fos expression after experimentally induced intraplantar inflammation. ${ }^{59}{ }^{60}$ Additionally, PGs appear to markedly contribute to the increased afferent discharge activity associated with ischaemia of the gastrointestinal tract. ${ }^{61}{ }^{62}$ The present study demonstrates that COX-2 inhibition can decrease spinal Fos expression induced by gut inflammation. This was demonstrated using pharmacological blockade of COX-2 synthase activity and by using COX $-2^{-/-}$mice, with both experimental manoeuvres decreasing the number of Fos positive neurones by $50 \%$. These data provide further evidence for the concept that COX-2 is an important emerging primary afferent modulator. ${ }^{63}$

$\mathrm{PGE}_{2}$ has been shown to have a direct effect on mesenteric afferents by interacting with EP receptor subtypes on the afferent receptive field nerve terminals. ${ }^{17}$ However, the mechanism by which COX-2 activity mediates an increase in primary afferent activity may not only be by a direct effect but could also be through potentiation of bradykinin induced activation of intestinal primary afferents. ${ }^{16}{ }^{18}$ Bradykinin is a pain producing peptide generated in tissues following inflammation that is known to stimulate primary afferents within the gastrointestinal tract. ${ }^{64}$ Recently it has been found that this response is also dependent on the presence of PGE. Inhibition of COX activity with naproxen significantly reduced the stimulatory effect of bradykinin on serosal afferents and direct application of $\mathrm{PGE}_{2}$ fully restored this response. ${ }^{16}$ Similarly, as COX-2 inhibition decreased the inflammatory infiltrate, the influence of leucocyte derived reactive intermediates such as superoxides and peroxynitrites would also be diminished and thus lessen their potential effects on altering primary afferent activity.

In conclusion, our study demonstrates for the first time that intestinal manipulation markedly increases spinal Fos expression for a prolonged period postoperatively in two rodent species. COX-2 plays a key role in this response, adding further to the evidence that COX-2 is a multifunctional neuronal modulator. We hypothesise that this may reflect primary afferent activation, initiating subsequently inhibitory motor reflexes to the gut, leading to postoperative intestinal gut dysfunction. Thus the present study shows that prostaglandins provide a crucial link between postoperative intestinal inflammatory mechanisms and activation of neuronal pathways in the pathogenesis of postoperative ileus.

\section{ACKNOWLEDGEMENTS}

This work was supported by the National Institutes of Health R01-GM58241, P50-GM- 53789, and RO1-DK54824. C Kreiss was supported by the Foundation for Digestive Health and Nutrition/ American Gastroenterological Association/AstraZeneca Fellowship/ Faculty Transition Award.

\section{Authors' affiliations}

C Kreiss, A J Baver, Division of Gastroenterology, Hepatology, and Nutrition, University of Pittsburgh Medical Center, Pittsburgh,

Pennsylvania 15261, USA

L A Birder, S Kiss, M M VanBibber, Department of Medicine, University of Pittsburgh Medical Center, Pittsburgh, Pennsylvania 15261, USA

\section{REFERENCES}

1 Holte K, Kehlet H. Postoperative ileus: a preventable event. Br J Surg 2000;87:1480-93. 
2 Holzer P, Lippe IT, Holzer-Petsche U. Inhibition of gastrointestinal transit due to surgical trauma or peritoneal irritation is reduced in capsaicin-treated rats. Gastroenterology 1986;91:360-3

3 Zittel TT, Meile T, Jehle EC, et al. Intraperitoneal capsaicin treatment reduces postoperative gastric ileus in awake rats. Langenbecks Arch Surg 2001;386:204-11.

4 Zittel TT, Lloyd KC, Rothenhofer I, et al. Calcitonin gene-related peptide and spinal afferents partly mediate postoperative colonic ileus in the rat. Surgery 1998;123:518-27.

5 Kalff JC, Schraut WH, Billiar TR, et al. Role of inducible nitric oxide synthase in postoperative intestinal smooth muscle dysfunction in rodents. Gastroenterology 2000;1 18:316-27.

6 Tanila H, Kauppila T, Taira T. Inhibition of intestinal motility and reversa of postlaparotomy ileus by selective alpha 2 -adrenergic drugs in the rat. Gastroenterology 1993;104:819-24.

7 De Winter BY, Boeckxstaens GE, De Man JG, et al. Effect of adrenergic and nitrergic blockade on experimental ileus in rats. $\mathrm{Br} J$ Pharmacol 1997; 120:464-8.

8 Kalff JC, Schwarz NT, Walgenbach KJ, et al. Leukocytes of the intestinal muscularis: their phenotype and isolation. J Leukoc Biol 1998;63:683-91.

9 Kalff JC, Schraut WH, Simmons RL, et al. Surgical manipulation of the gut elicits an intestinal muscularis inflammatory response resulting in postsurgical ileus. Ann Surg 1998;228:652-63.

10 Kalff JC, Hierholzer C, Tsukada K, et al. Hemorrhagic shock results in intestinal muscularis intercellular adhesion molecule (ICAM-1) expression neutrophil infiltration, and smooth muscle dysfunction. Arch Orthop Trauma Surg 1999;119:89-93.

11 Kalff JC, Carlos TM, Schraut WH, et al. Surgically induced leukocytic infiltrates within the rat intestinal muscularis mediate postoperative ileus. Gastroenterology 1999;117:378-87.

12 Schwarz NT, Kalff JC, Turler A, et al. Prostanoid production via COX-2 as a causative mechanism of rodent postoperative ileus. Gastroenterology 2001;121:1354-71.

13 Josephs MD, Cheng G, Ksontini R, et al. Products of cyclooxygenase-2 catalysis regulate postoperative bowel motility. I Surg Res 1999;86:50-4.

14 Nathan CF. Secretory products of macrophages. J Clin Invest 1987;79:319-26

15 Adelson DW, Wei JY, Kruger L. H2O2 sensitivity of afferent splanchnic C fiber units in vitro. J Neurophysiol 1996;76:371-80.

16 Maubach KA Grundy D. The role of prostaglandins in the bradykinin-induced activation of serosal afferents of the rat jejunum in vitro. J Physiol 1999;515:277-85.

17 Haupt W, Jiang W, Kreis ME, et al. Prostaglandin EP receptor subtypes have distinctive effects on jejunal afferent sensitivity in the rat. Gastroenterology 2000;1 19:1580-9.

18 Brunsden AM, Grundy D. Sensitization of visceral afferents to bradykinin in rat jejunum in vitro. J Physiol 1999:521:517-27.

19 Dubois RN, Abramson SB, Crofford L, et al. Cyclooxygenase in biology and disease. FASEB J 1998;12:1063-73.

20 Mitchell JA, Larkin S, Williams TJ. Cyclooxygenase-2: regulation and relevance in inflammation. Biochem Pharmacol 1995;50:1535-42.

21 Dragunow M, Faull R. The use of Fos as a metabolic marker in neuronal pathway tracing. J Neurosci Methods 1989;29:261-5.

22 Traub RJ, Sengupta JN, Gebhart GF. Differential Fos expression in the nucleus of the solitary tract and spinal cord following noxious gastric distention in the rat. Neuroscience 1996;74:873-84.

23 Traub RJ, Herdegen T, Gebhart GF. Differential expression of Fos and c-jun in two regions of the rat spinal cord following noxious colorectal distention. Neurosci Lett 1993;160:121-5.

24 Miampamba $\mathbf{M}$, Sharkey KA. Fos expression in the myenteric plexus, spinal cord and brainstem following injection of formalin in the rat colonic wall. J Auton Nerv Syst 1999;77: 140-51

25 Riendeau D, Percival MD, Boyce S, et al. Biochemical and pharmacological profile of a tetrasubstituted furanone as a highly selective COX-2 inhibitor. Br J Pharmacol 1997;121:105-17.

26 Dinchuk JE, Car BD, Focht RJ, et al. Renal abnormalities and an altered inflammatory response in mice lacking cyclooxygenase II. Nature 1995:378:406-9

27 Langenbach R, Morham SG, Tiano HF, et al. Prostaglandin synthase gene disruption in mice reduces arachidonic acid-induced inflammation and indomethacin-induced gastric ulceration. Cell 1995;83:483-92.

28 Morham SG, Langenbach R, Loftin CD, et al. Prostaglandin synthase 2 gene disruption causes severe renal pathology in the mouse. Cell 1995:83:473-82

29 Kalff JC, Buchholz BM, Eskandari MK, et al. Biphasic response to gut manipulation and temporal correlation of cellular infiltrates and muscle dysfunction in rat. Surgery 1999;126:498-509.

30 Birder LA, de Groat WC. Contribution of C-fiber afferent nerves and autonomic pathways in the urinary bladder to spinal Fos expression induced by bladder irritation. Somatosens Mot Res 1998:15:5-12.

31 Birder LA, de Groat WC. Increased Fos expression in spinal neurons after irritation of the lower urinary tract in the rat. J Neurosc 1992;12:4878-89.

32 Birder LA, de Groat WC. Induction of Fos expression in spinal neurons by nociceptive and nonnociceptive stimulation of LUT. Am J Physiol 1993;265:R326-33.
33 Lu Y, Westlund KN. Effects of baclofen on colon inflammation-induced Fos, CGRP and SP expression in spinal cord and brainstem. Brain Res 2001:889:118-30.

34 Birder LA, Roppolo JR, ladarola M, et al. Electrical stimulation of visceral afferent pathways in the pelvic nerve increases Fos in the rat lumbosacral spinal cord. Neurosci Letters 1991;129:193-6.

35 Althausen PL, Gupta MC, Benson DR, et al. The use of neostigmine to treat postoperative ileus in orthopedic spinal patients. J Spinal Dis 2001;14:541-5.

36 Birder LA, Roppolo JR, Erickson VL, et al. Increased Fos expression in spinal lumbosacral projection neurons and preganglionic neurons after irritation of the lower urinary tract in the rat. Brain Res 1999;834:55-65.

37 Hunt SP, Pini A, Evan G. Induction of Fos-like protein in spinal cord neurons following sensory stimulation. Nature 1987;328:632-4.

38 Bielefeldt K, Ozaki N, Gebhart GF. Mild gastritis alters voltage-sensitive sodium currents in gastric sensory neurons in rats. Gastroenterology 2002;122:752-61.

39 Gebhart GF. Visceral pain-peripheral sensitisation. Gut 2000;47/suppl 4):iv54-5.

40 Gebhart GF. Pathobiology of visceral pain: molecular mechanisms and therapeutic implications IV. Visceral afferent contributions to the pathobiology of visceral pain. Am J Physiol Gastrointest Liver Physiol 2000;278:G834-8

41 Morgan C, Nadelhaft I, deGroat WC. The distribution within the spinal cord of visceral primary afferent axons carried by the lumbar colonic nerve of the cat. Brain Res 1986:398:11-17.

42 Schwarz NT, Kalff JC, Turler A, et al. Selective jejunal manipulation causes postoperative pan-enteric inflammation and dysmotility. Gastroenterology 2003 (accepted for publication)

43 Graber JN, Schulte WJ, Condon RE, et al. Relationship of duration of postoperative ileus to extent and site of operative dissection. Surgery 1982;92:87-92.

44 Vizzard MA. Increased expression of spinal cord Fos protein induced by bladder stimulation after spinal cord injury. Am J Physiol Regul Integr Comp Physiol 2000;279:R295-305.

45 Abbadie C, Besson JM. c-fos expression in rat lumbar spinal cord during the development of adjuvant-induced arthritis. Neuroscience 1992;48:985-93.

46 Zittel TT, Reddy SN, Plourde V, et al. Role of spinal afferents and calcitonin gene- related peptide in the postoperative gastric ileus in anesthetized rats. Ann Surg 1994;219:79-87.

47 Sagrada A, Fargeas M, Bueno L. Involvement of alpha- 1 and alpha-2 adrenoceptors in the postlaparotomy intestinal motor disturbances in the rat. Gut 1987:28:955-9.

48 Traub RJ, Silva E, Gebhart GF, et al. Noxious colorectal distention induced-Fos protein in limbic brain structures in the rat. Neurosci Lett 1996;215: 165-8

49 Fiocchi C. Intestinal inflammation: a complex interplay of immune and nonimmune cell interactions. Am J Physiol Gastrointest Liver Physiol 1997:273:G769-75.

50 Laird JM, Olivar T, Lopez-Garcia JA, et al. Responses of rat spinal neurons to distension of inflamed colon: role of tachykinin NK2 receptors. Neuropharmacol 2001;40:696-701.

51 Sanders KM, Ross G. Effects of endogenous prostaglandin E on intestinal motility. Am J Physiol 1978;234:E204-8.

52 Thor P, Konturek JW, Konturek SJ, et al. Role of prostaglandins in control of intestinal motility. Am J Physiol 1985;248:G353-9.

53 Tollstrom T, Hellstrom PM, Johansson C, et al. Effects of prostaglandins E2 and F2 alpha on motility of small intestine in man. Dig Dis Sci 1988:33:552-7.

54 Sanders KM. Endogenous prostaglandin E and contractile activity of isolated ileal smooth muscle. Am J Physiol 1978;234:E209-12.

55 Maggi CA, Giuliani S, Conte B, et al. Prostanoids modulate reflex micturition by acting through capsaicin-sensitive afferents. Eur J Pharmacol 1988; 145:105-12.

56 Williams JA, Shacter E. Regulation of macrophage cytokine production by prostaglandin E2. Distinct roles of cyclooxygenase-1 and -2. J Biol Chem 1997:272:25693-9.

57 Hierholzer C, Kalff JC, Chakraborty A, et al. Impaired gut contractility following hemorrhagic shock is accompaied by IL-6 and G-CSF production and neutrophil infiltration. Dig Dis Sci 2001;46:230-41.

58 Tegeder I, Pfeilschifter J, Geisslinger G. Cyclooxygenase-independent actions of cyclooxygenase inhibitors. FASEB J 2001;15:2057-72.

59 Buritova J, Chapman V, Honore P, et al. Selective cyclooxygenase-2 inhibition reduces carrageenan oedema and associated spinal Fos expression in the rat. Brain Res 1996;715:217-20.

60 Saade NE, Lawand HF, Safieh-Garabedian B, et al. Thymulin induces Fos expression in the spinal cord of rats which is reversed by meloxicam and morphine. J Neuroimmunol 1999;97:16-24

61 Longhurst JC, Rotto DM, Kaufman MP, et al. Ischemically sensitive abdominal visceral afferents: response to cyclooxygenase blockade. Am J Physiol 1991;261:H2075-81.

62 Longhurst JC, Dittman LE. Hypoxia, bradykinin, and prostaglandins stimulate ischemically sensitive visceral afferents. Am J Physiol 1987;253:H556-67.

63 Bazan NG. COX-2 as a multifunctional neuronal modulator. Nat Med 2001;7:414-15.

64 Longhurst JC, Kaufman MP, Ordway GA, et al. Effects of bradykinin and capsaicin on endings of afferent fibers from abdominal visceral organs. Am J Physiol 1984;247:R552-9. 